\title{
Caractérisation hydro-climatique, analyse comparative des termes du bilan hydrologique du bassin versant d'Ambouli (République de Djibouti)
}

\author{
Golab Moussa Omar ${ }^{1,2}$, Jean-Emmanuel Paturel ${ }^{1}$, Christian Salles ${ }^{1}$, Gil Mahé ${ }^{1}$, and Mohamed Jalludin ${ }^{2}$ \\ ${ }^{1}$ HSM, Univ. Montpellier, CNRS, IRD, Montpellier, France \\ ${ }^{2}$ Centre d'Etudes et de Recherches de Djibouti, Institut des Sciences de la Terre, \\ Route de l'aeroport, Djibouti-ville, Djibouti
}

Correspondence: Golab Moussa Omar (golabmoussa@gmail.com)

Published: 16 November 2021

\begin{abstract}
Résumé. Le bassin versant exoréique de l'Oued Ambouli situé au sud-est de la République de Djibouti, s'étend sur plus de $794 \mathrm{~km}^{2}$ soit $3.5 \%$ de la surface du pays. Il est le plus grand bassin versant du district de Djibouti. Cet oued est soumis à des crues rares et brèves qui causent de lourds dégâts en vies humaines et économiques, accentués par l'expansion urbaine de la ville de Djibouti. Cette étude vise à calculer les différentes composantes du bilan hydrologique. Sur la période 2008-2013, ce bassin a reçu une pluie moyenne annuelle de $93.6 \mathrm{~mm}$; l'évapotranspiration réelle (ETR) sur le bassin versant est estimée à $79.6 \mathrm{~mm} / \mathrm{an}$ soit $85 \%$ des précipitations moyennes annuelles; l'écoulement total approche un volume de $6.5 \times 10^{6} \mathrm{~m}^{3} / \mathrm{an}$, soit $3.7 \mathrm{~mm} / \mathrm{an}$; par déduction, selon la formule du bilan hydrologique, l'infiltration totale est estimée à $10.3 \mathrm{~mm} / \mathrm{an}$. Ces résultats comparés aux résultats obtenus antérieurement, apparaissent comme très différents (principalement une baisse des valeurs). Les données disponibles sont très fragmentées dans le temps et ne couvrent que des périodes trop courtes pour pouvoir prétendre fournir des valeurs de référence pour un bilan hydrologique et permettre un suivi évolutif de celui-ci.
\end{abstract}

\section{Introduction}

La ville de Djibouti, capitale de la République de Djibouti, s'est développée sur le bassin versant de l'Oued Ambouli. Cet oued est à l'origine de crues rares et brèves avec de lourds dégâts en vie humaine et économiques qui sont accentués par l'expansion urbaine de la ville. Afin de contribuer à la prévention contre les risques d'inondations, qui surviennent de plus en plus fréquemment, et à la protection de la ressource en eau dans le bassin de l'Oued Ambouli, nous avons collecté toutes les données et tous les travaux disponibles qui permettent, dans un premier temps, d'établir un bilan hydrologique. Bien que d'une importance stratégique pour la ville de Djibouti, cet oued n'a fait l'objet que de rares suivis hydrologiques, sur des périodes très courtes et à des moments très différents de son développement sans qu'un suivi de son évolution ne puisse être établi. Cette étude permet de rassembler l'état (maigre) des connaissances sur les composantes du bilan hydrologique du bassin afin d'en faire une synthèse et d'en déduire des pistes de recherche scientifiques et/ou de données.

Nous avons rassemblé et analysé les données climatiques et hydrologiques disponibles auprès du Centre d'Etude et de Recherche de Djibouti sur la période 2008-2013 (CERD). Ces données ont permis d'établir un "instantané" du bilan hydrologique du bassin qui a, alors été, comparé aux estimations de ce bilan des travaux de la Coopération Hydrologique Allemande (CHA, 1982) et de Gamal-Eldin (1988). In fine, ces résultats seront les premiers éléments de guide pour la mise en place d'une stratégie holistique de compréhension du cycle de l'eau, depuis les eaux de surface jusqu'aux eaux souterraines. Ces premiers résultats nous permettront d'orienter la suite de nos travaux. 


\section{Presentation de la zone d'etude}

La République de Djibouti, d'une superficie de $23200 \mathrm{~km}^{2}$, présente un climat tropical aride qui se caractérise par l'irrégularité et la faiblesse des précipitations annuelles, avec des températures élevées durant toute l'année, l'absence de cours d'eau pérenne et une évaporation très intense. La saison fraiche, d'octobre à mars, présente des températures modérées (entre 22 et $30^{\circ} \mathrm{C}$ ) et une humidité relativement élevée. La saison chaude de juin à septembre est marquée par des températures entre 30 et $40^{\circ} \mathrm{C}$ et un vent sableux, sec et chaud (le Khamsin). La période intermédiaire (avril-mai) fait la transition entre saison fraiche et saison chaude.

Le bassin versant exoréique de l'Oued Ambouli, situé au sud-est de la République de Djibouti, s'étend sur plus de $794 \mathrm{~km}^{2}$ soit $3.5 \%$ de la surface du pays (Fig. 1). C'est le plus grand bassin versant du district de Djibouti qui accueille les deux tiers de la population de la République de Djibouti (570 000 habitants). Ce bassin versant, de par sa localisation géographique et ses ressources en eau souterraine, est d'une importance majeure pour le développement de la ville de Djibouti. En effet d'une part, le delta de l'oued dans l'Océan Indien constitue un vaste cône aplati prolongé par une presqu'̂̂le corallienne sur lequel est implantée la ville de Djibouti (SAFEGE, Consortium STDE, 2007), d'autre part, le soussol de la partie aval du bassin versant est constitué d'un épandage basaltique daté fin tertiaire / début quaternaire de plusieurs dizaines de mètres d'épaisseur qui contient une nappe aquifère abondante et de bonne qualité. Depuis plusieurs décennies, la quasi-totalité de l'eau nécessaire à l'alimentation de la ville de Djibouti est captée dans cet aquifère. A l'heure actuelle, le prélèvement annuel dépasse 13 millions de $\mathrm{m}^{3}$ (Houmed Gaba, 2009) L'augmentation de la population s'accompagne d'une croissance rapide des besoins qui entraîne une surexploitation et une dégradation de cette ressource.

\section{Données et methodes}

\subsection{Presentation des données}

Les données hydrométéorologiques (principalement pluviometrique) disponibles sur le bassin d'Ambouli sont très disparates dans le temps et dans l'espace (Figs. 1 et 2). L'équipement des capteurs pluviometriques du bassin s'est fait progressivement avec une interruption des observations sur la période 1990-2008. La plus longue série disponible est la pluviométrie enregistrée à la station météorologique de Djibouti-Aérodrome en continu depuis 1953. C'est à partir de cette station que l'ETP et L'ETR ont été calculées.

Les résultats produits par la CHA ont été obtenus sur la base de données mensuelles des stations limnimétriques d'Ambouli et Oueah et des stations pluviométriques d'Ambouli, Oueah et Arta collectées sur la période 1980 à 1982 (Fig. 2). Les mesures de débit antérieures à 1980 n'ont pas pu être utilisées car elles sont très incomplètes. Les stations sont installées de manière à ne mesurer que les hauteurs d'eau supérieures à $1 \mathrm{~m}$ dans le cours d'eau.

Les travaux de Gamal-Eldin (1988) se sont appuyés sur le même réseau d'observation que la CHA, complété par les séries pluviometriques observées sur la période 1983 à 1986.

C'est à partir de 2008, suite aux inondations dévastatrices de 1994 et 2004, que le CERD a renforcé le suivi hydrométéorologique du bassin d'Ambouli. Sur la période 20082013, le bassin était équipé de :

- 8 stations pluviométriques avec pour la plupart des données infra journalières (données numérisées).

- 1 station de mesure de la température qui est la station Aérodrome (Djibouti-aérodrome 1962-2018).

- 3 stations limnimétriques (Boulleh, Kalaloho et Oueah) avec des données infra journalières (données numérisées).

Les données disponibles pour chacune des études passées (CHA, 1982; Gamal-Eldin, 1988) et présentes, sont trop fragmentées dans le temps et ne couvrent que des périodes trop courtes pour pouvoir prétendre fournir des valeurs de référence.

\subsection{Méthodes de calcul des termes du bilan hydrologique}

Simple opération comptable, le bilan hydrologique vise à établir le bilan hydrique d'une unité hydrologique définie (le bassin versant) pendant une période de temps donné. Il s'écrit :

$$
P=Q+\mathrm{ETR}+\Delta R(u+h)
$$

La pluie $(P)$ qui tombe sur le bassin versant dans un laps de temps donné soit s'écoule $(Q)$, soit repart dans l'atmosphère par évapotranspiration (ETR), soit participe à la recharge des réserves en eau du sol (Ru) ou du sous-sol (Rh). Les variations de réserve peuvent être négatives et contribuer aux écoulements et/ou à l'évapotranspiration.

Pour déterminer la précipitation moyenne $P$ du bassin versant, on utilisé les trois méthodes d'interpolation les plus courantes sont :

- La méthode arithmétique qui est une méthode simple qui consiste à déterminer les moyennes arithmétiques entre stations.

- La méthode des isohyètes (L'Hote, 1993), méthode graphique qui se base sur l'interpolation des moyennes de précipitation,

- La méthode des polygones de Thiessen (Schumann, 1998), méthode qui consiste à effectuer la moyenne pondérée des précipitations observées dans les différentes stations pluviométriques du bassin versant en fonction de leur aire d'influence géométrique. 


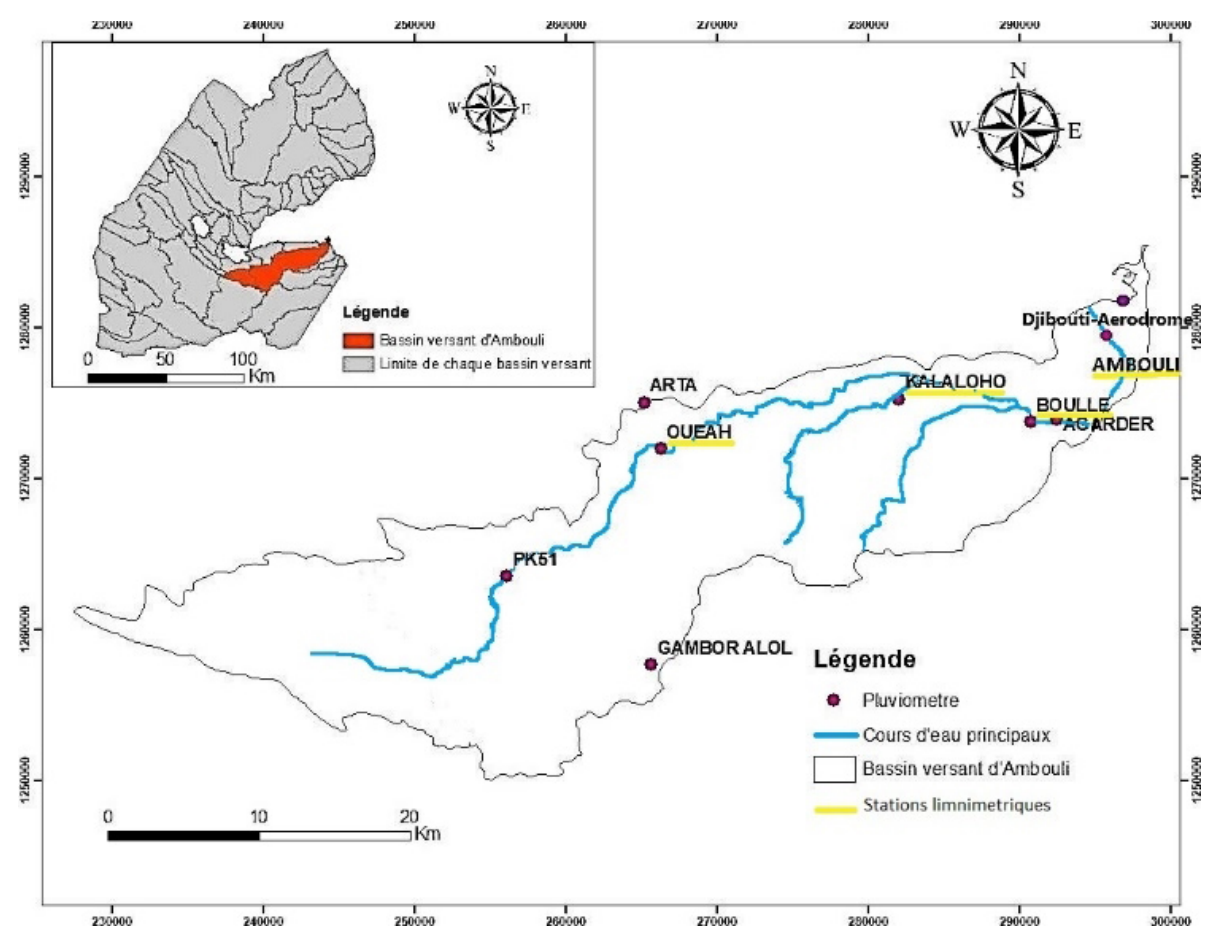

Figure 1. Bassin de l'Oued Ambouli et localisation des stations pluviométriques et limnimétriques (C) CERD).

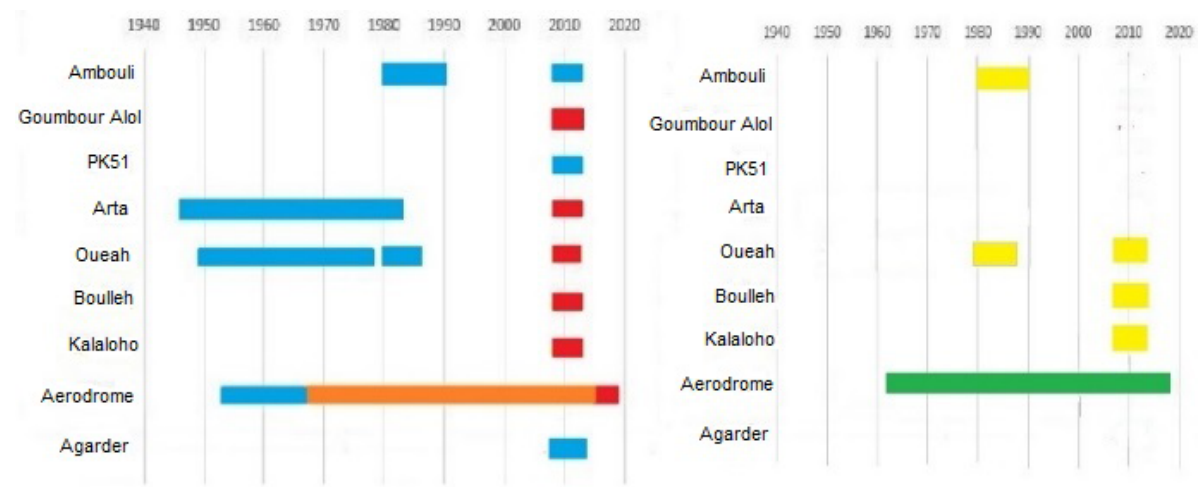

Figure 2. Données disponibles sur le bassin versant d'Ambouli. La Pluviométrie (Rouge : infra-journalier, Orange : journalier, Bleu : mensuel); la Limnimétrie (Jaune : infra-journalier); la Temperature (Vert : Journalier).

Le concept de l'évapotranspiration potentielle (ETP) a été introduit par Thornthwaite et Penman car l'évapotranspiration réelle est difficile à mesurer à l'échelle d'une parcelle ou d'une région. Pour déterminer l'évapotranspiration potentielle moyenne, les trois méthodes les plus courantes sont :

- La formule de Thornthwaite (Thornthwaite et Mather, 1955) qui n'utilise que la température et un indice thermique comme donnée climatique.

- La formule de Turc (Turc, 1961) qui fait intervenir plusieurs paramètres climatologiques (température, rayonnement et humidité).

- La formule de Penman simplifiée (Penman, 1963).
L'évapotranspiration réelle est en règle générale obtenue grâce à une réduction de l'évapotranspiration potentielle :

- La méthode du bilan de Thornthwaite (Thornthwaite et Mather, 1955) qui consiste à calculer l'ETR mensuelle à partir des précipitations moyennes mensuelles, l'ETP et la réserve facilement utilisable contenue dans le sol (RFU),

- La méthode de Turc (Turc, 1961) qui n'utilise que deux paramètres climatologiques, la température et la précipitation.

L'écoulement $Q$ est mesuré aux stations hydrologiques d'Ambouli et Oueah (CHA, 1982 et Gamal-Eldin, 1988) 
Tableau 1. Tableau comparatif des composantes du bilan hydrologique.

\begin{tabular}{|c|c|c|c|c|}
\hline & Méthode & $\begin{array}{l}\text { CHA } \\
(1980-1982)\end{array}$ & $\begin{array}{l}\text { Gamal-Eldin } \\
(1982-1986)\end{array}$ & $\begin{array}{l}\text { CERD } \\
(2008-2013)\end{array}$ \\
\hline \multirow{3}{*}{$\begin{array}{l}\text { Précipitation } \\
(\mathrm{mm} / \mathrm{an})\end{array}$} & Arithmétique & 176.5 & 104 & 91.7 \\
\hline & Thiessen & $\mathrm{ND}^{*}$ & ND & 94.7 \\
\hline & Isohyètes & 200.0 & ND & 94.4 \\
\hline \multirow{2}{*}{$\begin{array}{l}\text { Evapotranspiration } \\
\text { (mm/an) }\end{array}$} & Thornthwaite & 157 & 85 & 80 \\
\hline & Turc & ND & ND & 98 \\
\hline \multirow{2}{*}{$\begin{array}{l}\text { Ecoulement } \\
(\mathrm{mm} / \mathrm{an} \text { et } \%)\end{array}$} & Coeff. de & $11.3 \mathrm{~mm} / \mathrm{an}$ & $5.2 \mathrm{~mm} / \mathrm{an}$ & $3.7 \mathrm{~mm} / \mathrm{an}$ \\
\hline & ruissellement & $6 \%$ & $5 \%$ & $4 \%$ \\
\hline \multirow{2}{*}{$\begin{array}{l}\text { Infiltration } \\
(\mathrm{mm} / \mathrm{an} \text { et } \%)\end{array}$} & Déduite du bilan & $21.6 \mathrm{~mm} / \mathrm{an}$ & $11.4 \mathrm{~mm} / \mathrm{an}$ & $10.3 \mathrm{~mm} / \mathrm{an}$ \\
\hline & hydrologique & $11.5 \%$ & $11 \%$ & $11 \%$ \\
\hline Station & & Ambouli Oueah & Ambouli Oueah & 7 stations \\
\hline
\end{tabular}

* ND : non disponible.

et Boulleh, Kalaloho et Oueah pour les données du CERD (2008-2013).

L'infiltration $\Delta R$ est déduite à partir de la formule du bilan hydrologique et de la connaissance des 3 termes : $P, Q$ et ETR pour la CHA (1982) et Gamal-Eldin (1988). Les travaux de Jalludin et Razack (2008) basés sur la modélisation numérique de l'aquifère confirment les évaluations de l'infiltration obtenues par la CHA (1982) et Gamal-Eldin (1988).

\section{Analyse et comparaison des resultats issus des differentes methodes de calcul de chaque composante du bilan hydrologique}

\subsection{La pluie}

La CHA (1982) a utilisé deux méthodes afin de déterminer la précipitation moyenne annuelle, la méthode arithmétique $(176.5 \mathrm{~mm} / \mathrm{an})$ et la méthode des isohyètes $(200 \mathrm{~mm} / \mathrm{an})$.

Gamal-Eldin (1988), à partir des stations d'Ambouli, Oueah et Djibouti Aérodrome, a obtenu une précipitation moyenne annuelle de $104 \mathrm{~mm} / \mathrm{an}$ (Tableau 1) en utilisant la méthode arithmétique. L'écart avec le calcul précédent est important.

Sur la période 2008-2013, nous disposons d'observations plus fines et plus complètes sur la pluie (Fig. 3). Cependant, cette courte période de 5 années d'observation ne permet pas une représentativité fiable des moyennes et des extrêmes saisonniers. La pluie moyenne calculée selon les trois méthodes avoisine $94 \mathrm{~mm}$ (min-max). Elle est légèrement plus faible que la valeur calculée par Gamal-Eldin (1988). Nous constatons que les precipitations moyennes mensuelles des mois de juin et juillet sont très faibles, moins de $5 \mathrm{~mm}$ par mois (Fig. 3), contrairement aux mois de mars, avril et mai qui représentent plus de $55 \%$ de la pluviométrie annuelle. Nous remarquons également que pour cinq des huit stations analysées, les valeurs maximales des précipitations moyennes

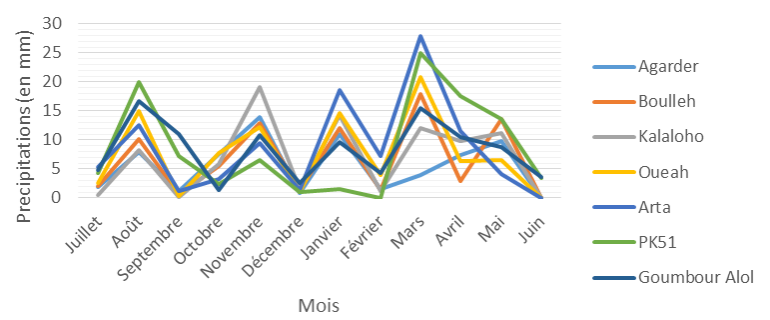

Figure 3. Variation des précipitations moyennes mensuelles dans le bassin versant d'Ambouli (2008-2013).

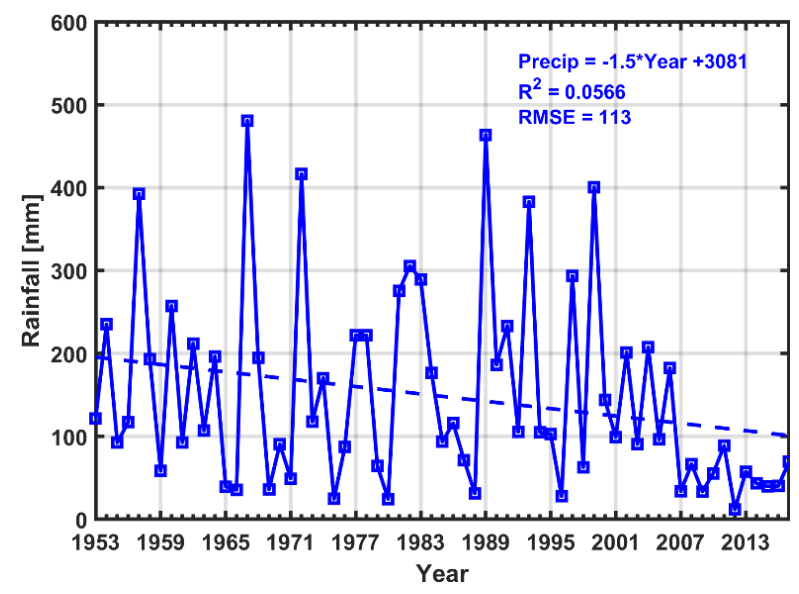

Figure 4. Evolution de la pluie annuelle de la station DjiboutiAérodrome (1953-2018).

mensuelles sont enregistrées au mois de mars et les valeurs minimales au mois de juin pour les sept stations. Nous remarquons enfin une forte irregularité spatiale des cumuls mensuels sur le bassin versant (Fig. 3).

Concernant les précipitations annuelles, le maximum est observé à la station de Goumbour Alol (avec 103 mm/an en 


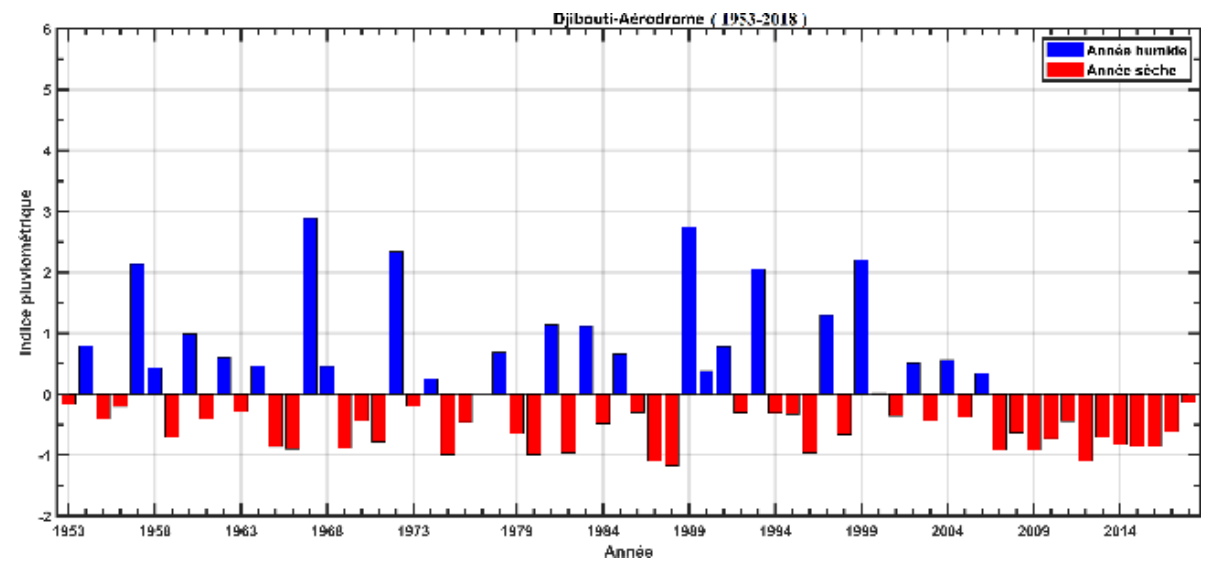

Figure 5. Anomalie pluviometrique (Indice de Lamb) de la station Djibouti aérodrome (1953-2018).

moyenne sur la période 2008-2013) et le minimum au niveau de la station Djibouti-Aérodrome (avec $55 \mathrm{~mm}$ ). Cette variation des précipitations dépend fortement de la localisation de la station climatologique : on constate que les précipitations augmentent avec l'altitude au niveau de la zone d'etude.

Concernant l'évolution de la pluviométrie annuelle au niveau de la station Djibouti-Aérodrome (1953-2018), l'analyse de la Fig. 4 montre une forte variabilité temporelle des données pluviométriques avec un maximum de $500 \mathrm{~mm}$ (en 1967) et un minimum de $11 \mathrm{~mm}$ (en 1987).

Cette variabilité interannuelle semble être moindre depuis une dizaine d'années avec une valeur de pluie annuelle inférieure à la moyenne annuelle sur la période 1953-2018 (Djama and Gascon, 1997). Nous constatons également une tendance à la baisse (Fig. 4) et une période sèche qui commence à partir de 2007 (Fig. 5). Cet indice pluviometrique de Lamb (Fig. 5) est la difference entre la precipitation annuelle et sa moyenne puis divisé par l'ecart de type de ce dernier.

\subsection{L'évapotranspiration}

La CHA a mesuré l'ETP aux stations de Djibouti Aérodrome et Arta. À la station Arta, l'ETP a été mesurée à l'aide de l'évaporomètre de Piche, alors qu'à la station DjiboutiAérodrome, elle a été mesurée sur un bac évaporatoire Classe A. Afin de rendre comparable ces données d'ETP, les ingénieurs de la CHA ont établi la relation suivante Eq. (1) :

$E_{\mathrm{pot}, \mathrm{BC}}=0.947 E_{\mathrm{pot}, \mathrm{piche}}-0.096$

$E_{\mathrm{pot}, \mathrm{BC}}$ : Evaporation du bac évaporatoire Classe A $(\mathrm{mm} / \mathrm{j})$. $E_{\mathrm{pot}, \text { piche }}:$ Evaporation mesurée par l'evaporimetre Piche $(\mathrm{mm} / \mathrm{j})$.

Après cette conversion des données de l'ETP, la CHA a utilisé la méthode du bilan de Thornthwaite (Thornthwaite et Mather, 1955) sur les stations Djibouti Aérodrome et Arta pour estimer une ETR moyenne annuelle à $157 \mathrm{~mm} / \mathrm{an}$.

Gamal-Eldin (1988) en utilisant la même méthode que la CHA sur la période 1980-1986 a obtenu une ETR moyenne annuelle de $85 \mathrm{~mm} / \mathrm{an}$ (Tableau 1). L'écart avec le calcul précédent est important en valeur absolue tout comme ce qui avait été observé sur les pluies.

L'ETP et l'ETR sont estimées au niveau des stations Djibouti Aérodrome et Arta qui toutes deux se trouvent légèrement en bordure de l'oued Ambouli. Les pluies etant très variables sur le bassin (Fig. 3), on peut s'interroger sur la représentativité de ces données utilisées par ces deux etudes pour l'élaboration des bilans hydrologiques.

Les données de 2008-2013 donnent une ETR moyenne annuelle de $80 \mathrm{~mm} /$ an (Tableau 1) en utilisant la méthode de bilan de Thornthwaite (Thornthwaite et Mather, 1955). La méthode de Turc (Turc, 1961) donne une ETR moyenne de $98.5 \mathrm{~mm} / \mathrm{an}$. On remarquera que cette valeur moyenne de l'ETR est supérieure à la précipitation moyenne annuelle $(93.6 \mathrm{~mm})$. De ce fait cette formule s'avère être inadéquate, mais en revanche ce résultat permet de constater que la valeur de l'ETR se rapproche beaucoup de celle de la précipitation annuelle moyenne.

Il ressort de cette analyse une grande variabilité de la valeur de l'ETR dans le temps : l'ETP obtenue à DjiboutiAérodrome avec la formule de Penman simplifiée est de $1800 \mathrm{~mm} / \mathrm{an}$. Lorsque nous comparons les valeurs calculées avec cette formule à l'evaporation mesurée nous constatons que ces valeurs sont proches contrairement aux autres methodes de calculs. Cette variabilité est principalement due au pas de temps mensuel (dans un climat aride) car il n'y a pas d'adéquation entre le régime des pluies (sur quelques jours de pluie seulement) et l'évapotranspiration car dans un pas de temps mensuel, on sous-estime en général les pluies efficaces.

\subsection{Ecoulement}

Pour évaluer le ruissellement produit par ce bassin versant, la CHA (1982) et Gamal-Eldin (1988) ont analysé les crues événement par événement et en ont déduit un coefficient d'écoulement moyen aux stations limnimétriques Ambouli 
et Oueah. En s'appuyant également sur une étude antérieure (Hauquin, 1978), la CHA (1982) a obtenu une valeur de coefficient d'ecoulement moyen de 6\%. Gamal-Eldin (1988), prenant en considération les études d'Hauquin (1978) et celle de CHA (1982) et les mesures de débits effectuées entre 1980 et 1986, parvient à une valeur de coefficient d'ecoulement de $5 \%$. Mais ce coefficient est très influencé par certains facteurs tels que l'imperméabilisation de la surface du sol et la précision des mesures de débit. Pour notre part, nous n'avons tenu compte que des coefficients d'ecoulement calculés par la CHA de 1980 à 1982 car ces calculs ne présentaient aucune lacune et avons obtenu une valeur de coefficient de ruissellement de $4 \%$.

Malgré une variabilité temporelle de ces données, ces valeurs de coefficient d'ecoulement sur une moyenne de valeurs évènementielles sont du même ordre de grandeur.

\subsection{Infiltration}

La CHA (1982) et Gamal-Eldin (1988) ont déterminé deux types d'infiltration, l'infiltration des eaux pluviales sur les surfaces des versants et l'infiltration dans les lits de l'oued. $\mathrm{Ne}$ disposant que de peu de données, l'association des deux types d'infiltration en infiltration totale a été nécessaire afin de déterminer le bilan hydrologique. La CHA (1982) et Gamal-Eldin (1988) ont effectué des mesures directes d'infiltration sur le bassin (Ambouli et Oueah) et noté la variabilité de l'ETR d'un mois à un autre. Le calcul de l'infiltration totale s'est fait par déduction de la formule du bilan hydrologique. Ainsi la CHA (1982) a obtenu une valeur de $21 \mathrm{~mm} / \mathrm{an}$ et Gamal-Eldin (1988) une valeur de $11.4 \mathrm{~mm} / \mathrm{an}$.

Nous avons pris en considération les études antérieures ainsi qu'une estimation de la recharge. Jalludin and Razack (2008) ont également tenu compte d'une forte incertitude à cause de l'effet du colmatage de la fissuration et de la porosité par les sédiments fins aux cours des périodes où l'eau stagne (Houmed-Gaba, 2009). L'infiltration a alors été estimée à $10.3 \mathrm{~mm} / \mathrm{an}$.

Il ressort de l'ensemble des travaux visant à estimer l'infiltration que c'est l'hétérogénéité du sol et la fluctuation mensuelle de l'ETR qui est à l'origine des écarts d'estimatiosn de l'infiltration mais relativement ces valeurs restent proches.

\section{Conclusion et perspectives}

La présente étude essaie d'approcher les termes du bilan hydrologique du bassin versant de l'Oued Ambouli à Djibouti et les compare à des travaux antérieurs (CHA et GamalEldin). Les données disponibles pour chacune des études passées et présentes sont trop fragmentées dans le temps et ne couvrent que des périodes trop courtes pour pouvoir prétendre servir de référence d'un bilan hydrologique. De plus, les caractéristiques statistiques entre les périodes des données montrent des differences importantes dont il va falloir tenir compte en développant une analyse de l'évolution du contexte climatique. Les données disponibles apparaissent insuffisantes en l'état actuel pour avoir une évaluation précise des termes du bilan et de leur évolution.

Cependant cette étude fournit quelques éléments guide de mise en place d'une stratégie holistique de compréhension du cycle de l'eau, depuis les eaux de surface jusqu' aux eaux souterraines. Elle fournit des éléments de connaissance nécessaires pour la protection de la ressource en eau et à la prévention contre les risques d'inondations, qui surviennent de plus en plus fréquemment dans le bassin de l'Oued Ambouli à Djibouti.

Suite à cette première étude, plusieurs travaux vont être menés afin de mieux approfondir les connaissances concernant le bilan hydrologique de ce bassin versant :

- Recherche de données climatiques alternatives, issues de grilles de données observées ou de produits satellitaires afin de mieux prendre en compte la variabilité spatiale et temporelle et l'évolution dans le temps.

- Analyse statistique de l'évolution de la pluviométrie (analyses des tendances ... ) et de la temperature et mise en relation avec la diminution de l'écoulement au sein du bassin versant et l'augmentation constatée des phénomènes d'inondation sur le bassin d'Ambouli.

- Critique, voire reprise, des évaluations antérieures, notamment en travaillant à des pas de temps plus fins (quotidien au lieu de mensuel) et à l'échelle de l'événement : saisie et/ou numérisation de données anciennes.

- Analyse de l'évolution de l'emprise urbaine dans la vallée et le delta de l'Oued Ambouli, pour suivre l'évolution de la vulnérabilité aux inondations.

- Mise en place d'un modèle hydrologique (afin de mieux comprendre le processus d'écoulement et les aléas associés) et, si possible, d'un modèle de prévention de crue.

- Mise en place d'un suivi continu et fiable des écoulements à une ou plusieurs stations.

Disponibilité des données. Les données utilisées sont issues des bases de données du Centre d'Etudes et de Recherches de Djibouti (CERD) et de l'Agence Météorologique Nationale (ANM) sont disponibles sur demande aupres de ces institutions.

Collaborateurs. GMO a fait la méthodologie, collecte de données, l'analyse de données, la rédaction-revue et l'édition. JEP a fait la méthodologie, l'analyse de données, la rédaction-revue et l'édition. CS a fait la méthodologie, l'analyse de données, la rédactionrevue et l'édition. GM a fait la méthodologie, l'analyse de données, la rédaction-revue et l'édition. $\mathrm{MJ}$ a fait la méthodologie, l'analyse de données, la rédaction-revue et l'édition. 
Intérêts concurrents. Les auteurs déclarent qu'ils n'ont aucun conflit d'intérêts.

Clause de non-responsabilité. Publisher's note : Copernicus Publications remains neutral with regard to jurisdictional claims in published maps and institutional affiliations.

Déclaration du numéro spécial. This article is part of the special issue "Hydrology of Large River Basins of Africa". It is a result of the 4th International Conference on the "Hydrology of the Great Rivers of Africa", Cotonou, Benin, 13-20 November 2021.

Financement. This research has been supported by the Campus France (Bourses doctorale du gouvernement francais).

\section{Références}

Coopération Hydrogéologique Allemande (CHA) : Inventaire et mises en valeur des ressources en eau de la République de Djibouti, 4 vol., République de Djibouti, 1982.

Djama, M. and Gascon, A. : "La Corne dans tous ses États", Cahiers d'Études africaines, 37, 146, 277-500, 1997.

Gamal-Eldin, H. A. : Etude de l'aquifère basaltique de la zone de captage de la ville de Djibouti, République de Djibouti, Thèse de doctorat, Univ. Albert-Ludwig de Fribourg, Fribourg-en-Brisgau, Allemagne Fédérale, 159 pp., 1988.

Hauquin, J. P. : La nappe côtière de la région de Djibouti. Géologie et Hydrogéologie, Thèse de Doctorat, Université de Bordeaux III, Bordeaux, France, 86 pp., 1978.

Houmed-Gaba, A. : Hydrogéologie des milieux volcaniques sous climat aride. Caractérisation sur site expérimental et modélisation numérique de l'aquifère basaltique de Djibouti (Corne de l'Afrique), PhD thesis, University of Poitiers, Poitiers, France, 220 pp., 2009.
Jalludin, M. and Razack, M. : Recharge simulation in stream bed alluviums overlying volcanic aquifer under semi-arid climate, Republic of Djibouti (Horn of Africa), in : Proceedings International Symposium SIHD, Djibouti, 14-17 December 2008, pp. 123-128, 2008.

L'Hote, Y. : Mesure et étude des précipitations en Hydrologie, Deust - Genie hydro sanitaire et aquacole, Univ. Montpellier, Montpellier, France, 38 pp., 1993.

Penman, H. L. : Vegetation and hydrology, Technical Communication, no. 53, Commonwealth Bureau of Soils, Harpenden, Commonwealth Agricultural Bureaux, Farham Royal, 1963.

SAFEGE, Consortium STDE : Projet d'Aménagement Intégré de L'Oued Ambouli. Etude d'identification des ouvrages de rétention des ruissellements et de recharge des nappes sur l'ensemble du bassin versant, Volume I et II, Republique de Djibouti, 127 pp., 2007.

Schumann, A. H. : Thiessen polygon, in : Encyclopedia of Hydrology and Lakes, Encyclopedia of Earth Science, Springer, Dordrecht, 1998.

Thornthwaite, C. W. and Mather, J. R. : The water balance, Climatology, 8, 5-86, 1955.

Turc, L. : Évaluation des besoins en eau d'irrigation, évapotranspiration potentielle, formule climatique simplifiée et mise à jour, Ann. Agron., 12, 13-49, 1961. 